\title{
Aufteilung einer ehemals einheitlichen Abteilung in zwei getrennte Kliniken Änderungskündigung eines Chefarztes ohne Verlustentschädigung kann wirksam sein
}

Ein Dauerbrenner bei Auseinandersetzungen zwischen Chefärzten und Kliniken sind aus organisatorischen Gründen vorgenommene Aufspaltungen existierender Abteilungen. Das Landesarbeitsgericht (LAG) Düsseldorf setzte sich mit seinem Urteil vom 30.10.2013 (Az.: 12 Sa 692/13) mit den hohen arbeitsrechtlichen Anforderungen an derartige Änderungskündigungen auseinander.

\section{Der Fall}

Der Kläger war auf Grundlage eines Dienstvertrages aus dem Jahr 1998 im Krankenhaus der Beklagten als Chefarzt der Klinik für Innere Medizin beschäftigt. Im Rahmen seines Dienstvertrages wurde ihm die Verantwortung für die medizinische Versorgung der Kranken in seiner Abteilung übertragen, wozu auch die Versorgung von Patienten mit führenden internistischen Krankheitsbildern auf der Intensivstation gehören sollte. Die Innere Abteilung war als eine Abteilung konzipiert, die die Allgemeine Innere Medizin verbunden mit den Schwerpunkten Gastroenterologie, Kardiologie und Diabetolo- gie umfasste. Des Weiteren wurde dem Chefarzt ein privates Liquidationsrecht eingeräumt. Im Übrigen sah der Dienstvertrag eine sogenannte Entwicklungsklausel vor, wonach sich der Träger das Recht vorbehielt, jederzeit selbstständige Fachabteilungen, jedoch nicht solche der gleichen Fachrichtung, oder Institute neu einzurichten oder abzutrennen und dafür weitere Abteilungsärzte einzustellen oder Belegärzte zuzulassen sowie neue Institutsleistungen zu erbringen. Der Klinikträger behielt sich weiterhin das Recht vor, die Bettenzahl der Abteilungen zu ändern, Behandlungseinrichtungen $\mathrm{zu}$ ändern, aufzulösen oder neu einzurich-

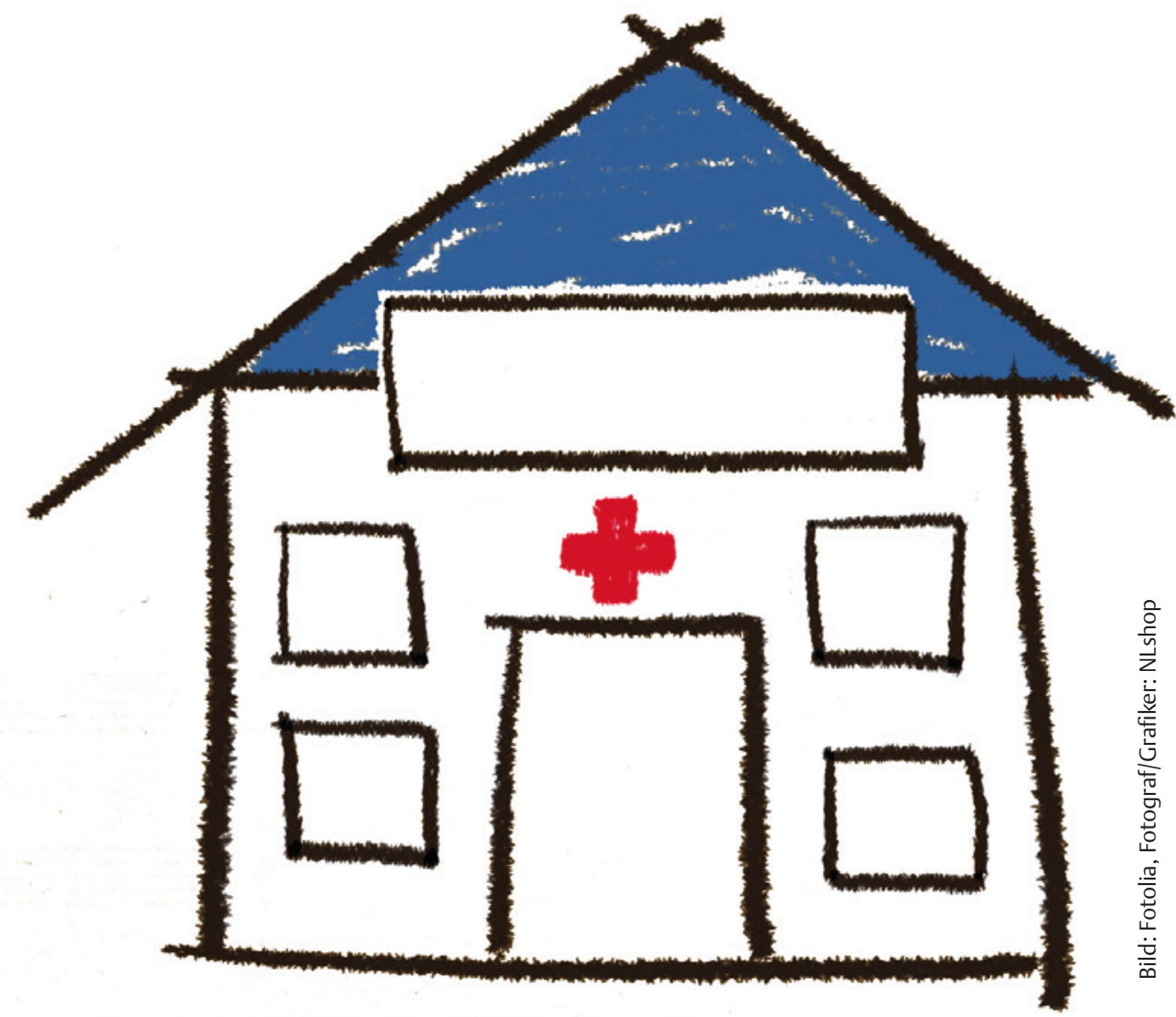

ten. Nach der Entwicklungsklausel war ein Ersatzanspruch des Arztes bei diesen Maßnahmen ausgeschlossen.

Die Beklagte wollte eine weitere Abteilung für Innere Medizin mit Schwerpunkt Kardiologie und konservativer Intensivmedizin einrichten, worüber sie auch mit dem Kläger verhandelte. Der Chefarzt widersetzte sich dieser Verselbstständigung der Abteilung grundsätzlich nicht, eine Einigung zu der von ihm geforderten finanziellen Entschädigung konnte jedoch nicht erzielt werden. Zum 01.01.2011 erfolgte die organisatorische Umsetzung der geplanten eigenständigen Abteilung mit einem weiteren Chefarzt, der über die Zusatzweiterbildung „Intensivmedizin“ verfügte.

\section{Zwei erfolglose}

\section{Änderungskündigungen}

Nach 2 zunächst erfolglosen Änderungskündigungen im Jahr 2010 bzw. 2011 traf die Gesellschafterversammlung der Klinik durch Beschluss die Entscheidung, weiterhin die bestehende Klinik für Innere Medizin aufzuspalten, indem die Klinik des Klägers auf die Schwerpunkte Gastroenterologie und Diabetologie begrenzt und eine neue Klinik mit den Schwerpunkten Kardiologie und konservative Intensivmedizin eingerichtet wird, die weiterhin vom Chefarzt Dr. W. geleitet werden sollte. Der Beschluss sah auch vor, dies gegenüber dem Kläger mit erneuter Änderungskündigung umzusetzen. Im Juni 2012 sprach daher die Beklagte eine erneute Änderungskündigung zum Jahresende aus. In der Änderungskündigung wurde dem Chefarzt mitgeteilt, dass er in Abänderung von $\S 1$ des Dienstvertrages ab dem 01.01.2013 als leitender Abteilungsarzt für alle Leistungen der Inneren Medizin zuständig sei, die nicht der bereits zum 01.01.2011 eingerichteten medizinischen Klinik II, die durch Herrn Dr. W. als leitenden Abteilungsarzt geleitet wird, zuzuordnen sind. Für die Abgrenzung der Behandlungsfälle wurden ausführliche Regelungen in die Änderungskündigung aufgenommen. Das dem Chef- 
arzt eingeräumte Liquidationsrecht wurde durch die Änderungskündigung ab dem 01.01.2013 auf die Leistungen der medizinischen Klinik I beschränkt.

\section{Chefarzt mit}

Änderungsschutzklage erfolglos

Der betroffene Chefarzt nahm diese Änderungskündigung rechtzeitig unter Vorbehalt an und erhob Änderungsschutzklage. Er wollte erreichen, dass festgestellt wird, dass die Änderungskündigung sozial ungerechtfertigt und rechtsunwirksam ist. Hilfsweise beantragte er, festzustellen, dass ihm ein Ersatzanspruch hinsichtlich der Leistungen zusteht, die er aufgrund der Änderungskündigung nicht mehr liquidieren kann. Das Arbeitsgericht gab zwar in erster Instanz seiner Klage statt. Nach Auffassung des Arbeitsgerichts war die Änderungskündigung unverhältnismäßig, weil dem Kläger die intensivmedizinische Behandlung seiner Patienten ohne Grund entzogen worden sei, zumal er diese in der Vergangenheit ohne Beanstandung ausgeübt habe. Hiergegen ging die Klinik jedoch in Berufung und hatte damit letztlich Erfolg. Nach Auffassung des LAG Düsseldorf war der Änderungsschutzantrag des Chefarztes unbegründet. Das Gericht stellte unter anderem fest, dass die Entwicklungsklausel des Arbeitsvertrages der Änderung der Arbeitsbedingungen nicht entgegenstünde. Nach Auffassung des Gerichts lagen dringende betriebliche Gründe im Sinne des Kündigungsschutzgesetzes (KSchG) vor. Die Änderungen der Arbeitsbedingungen seien auch hinreichend bestimmt gewesen.

Das LAG entschied: Eine betriebsbedingte Änderungskündigung sei im Sinne des Kündigungsschutzgesetzes sozial gerechtfertigt, wenn sich der Arbeitgeber bei Vorliegen eines Kündigungsschutzgrundes darauf beschränkt habe, solche Änderungen anzubieten, die der Arbeitnehmer billigerweise hinnehmen müsse. In diesem Rahmen sei zu prüfen, ob ein Beschäftigungsbedürfnis für den Arbeitnehmer zu den bisherigen Vertragsbedingungen entfallen sei und dem Arbeitnehmer bei der Anwendung des Verhältnismäßigkeitsgrundsatzes die am wenigsten beeinträchtigende Änderung angeboten worden sei. Dieser Maßstab gelte unabhängig davon, ob der Arbeitnehmer das Änderungsangebot ablehnt oder unter Vorbehalt angenommen habe.
Für jede Änderung muss eigenes

Bedürfnis vorliegen

Werden mit einer Änderungskündigung mehrere Änderungen vorgenommen, so müsse grundsätzlich für jede Änderung ein dringendes betriebliches Bedürfnis gegeben sein, so das LAG weiter. Die notwendigen dringenden betrieblichen Erfordernisse setzten voraus, dass das Bedürfnis für die Weiterbeschäftigung des Arbeitsnehmers im Betrieb zu den bisherigen Bedingungen entfallen sei. Dies könne auf einer unternehmerischen Entscheidung zur Umstrukturierung des gesamten oder von Teilen eines Betriebs bzw. einer Dienststelle oder einzelner Arbeitsplätze beruhen, von der aus das Anforderungsprofil der nach Umstrukturierung verbleibenden Arbeitsplätze erfasst werden könne, so die Richter unter Bezug auf verschiedene Bundesarbeitsgerichtsurteile.

\section{Keine Pflicht des Arbeitsgebers zur Beibehaltung bestehender Strukturen}

Es gehöre zum Kern der unternehmerischen Freiheit, die betriebliche Organisation zu gestalten und festzulegen, an welchem Standort welche arbeitstechnischen Zwecke und Ziele verfolgt werden sollen. Der gesetzliche Kündigungsschutz verpflichte den Arbeitgeber nicht, eine bestimmte betriebliche Organisationsstruktur beizubehalten. Zu diesem Entscheidungsspielraum des Arbeitgebers zähle grundsätzlich auch die Befugnis, den Inhalt der Arbeitsaufgaben und damit das Anforderungsprofil des Arbeitsplatzes zu bestimmen. Außerdem unterliege es seiner freien Entscheidung, die Zahl der Arbeitskräfte zu bestimmen, mit denen eine $\mathrm{Ar}$ beitsaufgabe - zukünftig - erledigt werden solle. Die beklagte Klinik habe ihre bisherige Abteilung der Inneren Medizin in die beiden Abteilungen der medizinischen Klinik I und II aufgeteilt mit der Folge, dass es eines Chefarztes für jede Klinik bedurfte, wobei jeweils unterschiedliche Schwerpunkte gebildet worden seien. Diese tatsächliche Trennung habe die Beklagte ab dem 01.01.2011 auch umgesetzt. Der Vortrag des Chefarztes ziele vor allem darauf $a b$, dass die vorgenommenen Änderungen über die Umsetzung der Organisationsentscheidung hinausgingen und es an einer Entschädigung für die entgangenen Liquidationserlöse fehle. Der tatsächlichen Aufteilung der beiden Kliniken sei der Kläger letztlich nicht entgegengetreten. 
Kein Rechtsmissbrauch ersichtlich Anhaltspunkte für eine rechtsmissbräuchliche Organisationsentscheidung seien nicht ersichtlich. Da die Änderungen der Organisationsstruktur tatsächlich umgesetzt worden seien, sei die Vermutung berechtigt, dass die Organisationsentscheidung aus sachlichen Gründen erfolgt sei und kein Rechtsmissbrauch vorliege. Des Weiteren stellt die Kammer klar, dass die Änderung der Arbeitsbedingungen in einem dringenden betrieblichen Erfordernis begründet sei. Grundsätzlich unterliege nämlich die Gestaltung des Anforderungsprofils eines Arbeitsplatzes der freien Unternehmerentscheidung. Gestalte der Arbeitgeber das Stellenprofil für Arbeitsplätze um, die bereits mit langjährig beschäftigten $\mathrm{Ar}$ beitnehmern besetzt seien, müsse er darlegen, dass es sich bei einer neuen Anforderung nicht nur um eine „wünschenswerte Voraussetzung“ für die Tätigkeit handelt, sondern um ein nachvollziehbares, arbeitsplatzbezogenes Kriterium für eine Stellenprofilierung. Die Entscheidung zur (neuen) Stellenprofilierung müsse im Zusammenhang mit einer organisatorischen Maßnahme stehen, nach deren Durchführung sich die bisherigen Anforderungen an den Stelleninhaber änderten. Diese Voraussetzungen seien hier zur Überzeugung der Kammer erfüllt. Es liege ein nachvollziehbares, arbeitsplatzbezogenes Kriterium für eine Stellenprofilierung vor. Nach Auffassung der Richter obliege es auch ohne eine Entscheidung über die fehlende Zusatzbezeichnung des Klägers der unternehmerischen Freiheit und Freiheit, das Anforderungsprofil arbeitsplatzbezogen nachvollziehbar zu ändern, wenn der Arbeitgeber sich dafür entscheide, die Patienten der Inneren Medizin in der primären Verantwortung von einem Chefarzt mit der Zusatzausbildung Intensivmedizin behandeln zu lassen, der als fachlicher Leiter der Abteilung die Letztverantwortlichkeit trägt.

\section{Verhältnismäßigkeitsgrundsatz gewahrt}

Nach Auffassung der Richter waren die vorgenommenen Änderungen durch die streitige Änderungskündigung auch verhältnismäßig. Es war zu prüfen, ob der Arbeitnehmer eine ihm vorgeschlagene Änderung billigerweise hinnehmen muss. Dies richtet sich nach dem Verhältnismäßigkeitsgrundsatz. Die Änderungen müssen geeignet und erforderlich sein, um den Inhalt des Arbeitsvertrages den geänderten Beschäftigungsmöglichkeiten anzupassen. Diese Voraussetzungen müssen für alle Vertragsänderungen vorliegen. Ausgangspunkt ist dabei die bisherige vertragliche Regelung, d. h. die angebotenen Änderungen dürfen sich nicht weiter vom Inhalt des bisherigen Arbeitsverhältnisses entfernen, als sie zur Erreichung des angestrebten Ziels erforderlich sind. Diese Voraussetzungen sah die Kammer als erfüllt an. Dies treffe zunächst für die inhaltlichen Festlegungen der Tätigkeiten zu, da (im Rahmen der dritten Änderungskündigung!) die erforderliche Abgrenzung der Tätigkeiten und Behandlungsfälle mit genauer Beschreibung der Zuständigkeiten nun erfolgt sei. Aber auch die Änderung des Liquidationsrechtes und damit die einhergehende Beschränkung der Nebentätigkeitserlaubnis waren nach Auffassung der Richter wirksam. Zwar sahen die Richter, dass hier mit dem Eingriff in das Liquidationsrecht auch Bestandteile des Arbeitsvertrages angegriffen wurden. Im Regelfall stelle die bloße tarifliche Vergütung ohne zusätzliche Einnahmemöglichkeit aus dem Liquidationsrecht keine angemessene Honorierung des Chefarztes dar. Ob ein Regelfall, bei dem dem Liquidationsrecht des Arztes Gegenleistungscharakter zukäme, vorliege, sei immer eine Frage der zwischen den Parteien getroffenen Vereinbarung. Im konkreten Fall läge ein solcher Fall vor. Das Liquidationsrecht knüpft zur Überzeugung der Kammer im konkreten Fall aber gerade an die Tätigkeit als Chefarzt in einem bestimmten Bereich an. So wird dem Kläger im Rahmen des Arbeitsvertrages gestattet, die erbrachten wahlärztlichen Leistungen seiner Abteilung gesondert privat abzurechnen.

\section{Beschränkung der Abteilung rechtfertigt Beschränkung des Liquidationsrechtes}

Wenn aber seine Abteilung eingeschränkt wird, kann er in diesem Bereich keine wahlärztlichen Leistungen mehr erbringen und auch nicht mehr liquidieren. Entgegen der Ansicht des Klägers sei es zur Wirksamkeit der Änderungskündigung nicht erforderlich, etwaige Verluste aufgrund der Einschränkung des Liquidationsrechtes auszugleichen. Vielmehr folge die Beschränkung des Liquidationsrechtes auf die Leistungen der medizinischen Klinik I wie bei einer Tarifautomatik der
Organisationsänderung. Ist der Kläger nur noch Leiter der medizinischen Klinik I, könne er nur noch deren Leistungen wahlärztlich abrechnen bzw. liquidieren. Letztlich werde die Organisationsänderung insoweit nur nachvollzogen. Das Liquidationsrecht werde dem Kläger auch nicht etwa vollständig entzogen. Es verbleibe ihm innerhalb seiner Abteilung. Das Liquidationsrecht knüpfte nach dem Arbeitsvertrag an die bisherige Leitung der Abteilung der Inneren Medizin an. Werde diese - wie vorgenommen - aufgespalten, gelte das Gleiche für das Liquidationsrecht. Die Entwicklungsklausel befasse sich nur mit der Frage des Direktionsrechtes und schließe insoweit Ersatzansprüche aus. Die Frage der Änderungskündigung sei nicht geregelt, sodass aus dem Umkehrschluss der Klausel auch keine Entschädigungspflicht abgeleitet werden könne. Abschließend stellten die Richter fest, dass das mit der Kündigung unterbreitete Änderungsangebot auch dem erforderlichen Gebot der hinreichenden Bestimmtheit entsprach. Es entsprach den Anforderungen, dass dem Angebot zweifelsfrei entnommen werden kann, welche Arbeitsbedingungen zukünftig gelten sollen.

\section{Fazit}

Auch nach dieser Entscheidung des LAG Düsseldorf wird es zukünftig weitere Auseinandersetzungen bei Aufspaltung bestehender Abteilungen geben, da es letztlich immer auf den Einzelfall ankommt. Von erheblicher Bedeutung ist dabei die Formulierung der Entwicklungsklausel im Arbeitsvertrag, auf die von beiden Seiten bereits beim Abschluss des Vertrages besonderes Augenmerk gelegt werden sollte. Wie der Fall (mit 2 vorausgegangenen erfolglosen Änderungskündigungen) zeigt, müssen Änderungskündigungen inhaltlich erhebliche Anforderungen erfüllen.

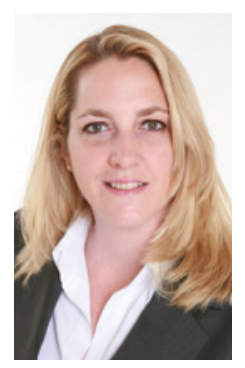

Korrespondenz Dr. iur. Isabel Häser Rechtsanwältin ECOVIS Lüdemann Wildfeuer \& Partner Sonnenstr. 9 80331 München www.ecovis.com 